\title{
The extended Mittag-Leffler function and its properties
}

\section{Mehmet Ali Özarslan and Banu Yılmaz}

"Correspondence:

banu.yilmaz@emu.edu.tr

Eastern Mediterranean University,

Famagusta, North Cyprus via

Mersin 10, Turkey

\begin{abstract}
In this paper, we present the extended Mittag-Leffler functions by using the extended Beta functions (Chaudhry et al. in Appl. Math. Comput. 159:589-602, 2004) and obtain some integral representations of them. The Mellin transform of these functions is given in terms of generalized Wright hypergeometric functions. Furthermore, we show that the extended fractional derivative (Özarslan and Özergin in Math. Comput. Model. 52:1825-1833, 2010) of the usual Mittag-Leffler function gives the extended Mittag-Leffler function. Finally, we present some relationships between these functions and the Laguerre polynomials and Whittaker functions.
\end{abstract}

Keywords: Mittag-Leffler; extended Beta functions; fractional derivative; Mellin transform; Laguerre polynomials; Whittaker functions; Wright generalized hypergeometric functions

\section{Introduction}

Fractional differential equations have been an active research area during the past few decades and they occur in many applications of physics and engineering. The MittagLeffler function appears as the solution of fractional order differential equations and fractional order integral equations. Some applications of the Mittag-Leffler function are as follows: studies of the kinetic equation, the telegraph equation [1], random walks, Levy flights, superdiffuse transport, and complex systems. Besides this, the Mittag-Leffler function appears in the solution of certain boundary value problems involving fractional integro-differential equations of Volterra type [2]. It has applications in applied problems, such as fluid flow, rheology, diffusive transport akin to diffusion, electric networks, probability, and statistical distribution theory. Various properties of the Mittag-Leffler functions were presented and surveyed in [3]. Furthermore, a different variant of the Mittag-Leffler function has been investigated in [4].

Let us start with giving the historical background of the Mittag-Leffler functions. The function $E_{\alpha}(z)$,

$$
E_{\alpha}(z)=\sum_{k=0}^{\infty} \frac{z^{k}}{\Gamma(\alpha k+1)}
$$

was defined and studied by Mittag-Leffler in the year 1903 in [5-7]. It is a direct generalization of the exponential series, since, for $\alpha=1$, we have the exponential function. The

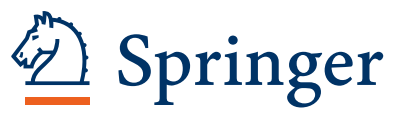

๑2014 Özarslan and Yılmaz; licensee Springer. This is an Open Access article distributed under the terms of the Creative Commons Attribution License (http://creativecommons.org/licenses/by/2.0), which permits unrestricted use, distribution, and reproduction in any medium, provided the original work is properly cited. 
function defined by

$$
E_{\alpha, \beta}(z)=\sum_{k=0}^{\infty} \frac{z^{k}}{\Gamma(\alpha k+\beta)}
$$

gives a generalization of equation (1). This generalization was studied by Wiman in 1905 [8, 9], Agarwal in 1953, and Humbert and Agarwal [10, 11] in 1953. Afterward, Prabhakar [12] introduced the generalized Mittag-Leffler function by

$$
E_{\beta, \gamma}^{\delta}(z):=\sum_{n=0}^{\infty} \frac{(\delta)_{n}}{\Gamma(\beta n+\gamma)} \frac{z^{n}}{n !}
$$

where $\beta, \gamma, \delta \in \mathbb{C}$ with $\Re(\beta)>0$. For $\delta=1$, it reduces to the Mittag-Leffler function given in equation (2). Some of the properties of the generalized Mittag-Leffler function such as the Mellin transform, the inverse Mellin transform, and differentiation were given in [13]. On the other hand, monotony of the Mittag-Leffler function was given in [14].

In this paper, we extend the Mittag-Leffler function $E_{\alpha, \beta}^{\gamma}(z)$ in the following way. Since

$$
E_{\alpha, \beta}^{\gamma}(z)=\sum_{k=0}^{\infty} \frac{(\gamma)_{k}}{\Gamma(\alpha k+\beta)} \frac{(c)_{k}}{(c)_{k}} \frac{z^{k}}{k !}
$$

using the fact that

$$
\frac{(\gamma)_{k}}{(c)_{k}}=\frac{B(\gamma+k, c-\gamma)}{B(\gamma, c-\gamma)}
$$

we extend the Mittag-Leffler function as follows:

$$
\begin{gathered}
E_{\alpha, \beta}^{(\gamma ; c)}(z ; p):=\sum_{k=0}^{\infty} \frac{B_{p}(\gamma+k, c-\gamma)}{B(\gamma, c-\gamma)} \frac{(c)_{k}}{\Gamma(\alpha k+\beta)} \frac{z^{k}}{k !} \\
(p \geq 0 ; \operatorname{Re}(c)>\operatorname{Re}(\gamma)>0),
\end{gathered}
$$

where for $B_{p}(x, y)$ we have

$$
B_{p}(x, y)=\int_{0}^{1} t^{x-1}(1-t)^{y-1} e^{-\frac{p}{t(1-t)}} d t \quad(\operatorname{Re}(p)>0, \operatorname{Re}(x)>0, \operatorname{Re}(y)>0),
$$

the extended Euler's Beta function defined in [15] (see also [16]).

The organization of the paper is as follows: In Section 2, we give an integral representations of the extended Mittag-Leffler function in terms of Prabhakar's Mittag-Leffler function and in terms of known elementary functions. The Mellin transform of the extended Mittag-Leffler function is obtained by means of the generalized Wright hypergeometric function [17]. In Section 3, we obtain fractional derivative representations of the extended Mittag-Leffler function and give some derivative formulas. In Section 4, we obtain the relationship between the extended Mittag-Leffler function and simple Laguerre polynomials and Whittaker's functions. 


\section{Some properties of the extended Mittag-Leffler function}

We begin with the following theorem, which gives the integral representation of the extended Mittag-Leffler function.

Theorem 1 (Integral representation) For the extended Mittag-Leffler function, we have

$$
E_{\alpha, \beta}^{(\gamma ; c)}(z ; p)=\frac{1}{B(\gamma, c-\gamma)} \int_{0}^{1} t^{\gamma-1}(1-t)^{c-\gamma-1} e^{-\frac{p}{t(1-t)}} E_{\alpha, \beta}^{(c)}(t z) d t
$$

where $p \geq 0, \operatorname{Re}(c)>\operatorname{Re}(\gamma)>0, \operatorname{Re}(\alpha)>0, \operatorname{Re}(\beta)>0$.

Proof Using equation (5) in equation (4), we get

$$
E_{\alpha, \beta}^{(\gamma ; c)}(z ; p)=\sum_{k=0}^{\infty}\left\{\int_{0}^{1} t^{\gamma+k-1}(1-t)^{c-\gamma-1} e^{-\frac{p}{t(1-t)}} d t\right\} \frac{(c)_{k}}{B(\gamma, c-\gamma)} \frac{z^{k}}{\Gamma(\alpha k+\beta) k !} .
$$

Interchanging the order of summation and integration in equation (7), which is guaranteed under the assumptions given in the statement of the theorem, we get

$$
\begin{aligned}
& E_{\alpha, \beta}^{(\gamma ; c)}(z ; p) \\
& \quad=\int_{0}^{1} t^{\gamma-1}(1-t)^{c-\gamma-1} e^{-\frac{p}{t(1-t)}} \sum_{k=0}^{\infty} \frac{(c)_{k}}{B(\gamma, c-\gamma)} \frac{(t z)^{k}}{\Gamma(\alpha k+\beta) k !} d t .
\end{aligned}
$$

Using equation (3) in equation (8), we get the desired result.

Corollary 2 Note that, taking $t=\frac{u}{1+u}$ in Theorem 1 , we get

$$
\begin{aligned}
& E_{\alpha, \beta}^{(\gamma ; c)}(z ; p) \\
& \quad=\frac{1}{B(\gamma, c-\gamma)} \int_{0}^{\infty} \frac{u^{\gamma-1}}{(u+1)^{c}} e^{-\frac{p(1+u)^{2}}{u}} E_{\alpha, \beta}^{(c)}\left(\frac{u z}{1+u}\right) d u .
\end{aligned}
$$

Corollary 3 Taking $t=\sin ^{2} \theta$ in the Theorem 1 , we get the following integral representation:

$$
\begin{aligned}
& E_{\alpha, \beta}^{(\gamma ; c)}(z ; p) \\
& =\frac{1}{B(\gamma, c-\gamma)}\left[2 \int_{0}^{\frac{\pi}{2}} \sin ^{2 \gamma-1} \theta \cos ^{2 c-2 \gamma-1} \theta e^{-\frac{p}{\sin ^{2} \theta \cos ^{2} \theta}}\right] \\
& \quad \times E_{\alpha, \beta}^{(c)}\left(z \sin ^{2} \theta\right) d \theta .
\end{aligned}
$$

Now, using the definition of Prabhakar's Mittag-Leffler's function, Bayram and Kurulay obtained the recurrence formula [13]

$$
E_{\alpha, \beta}^{(c)}(t z)=\beta E_{\alpha, \beta+1}^{(c)}(t z)+\alpha z \frac{d}{d z} E_{\alpha, \beta+1}^{(c)}(t z) .
$$

Inserting the above recurrence relation into equation (6), we get the following recurrence relation for the extended Mittag-Leffler's function. 
Corollary 4 (Recurrence relation) For the extended Mittag-Leffler function, we get

$$
E_{\alpha, \beta}^{(\gamma ; c)}(z ; p)=\beta E_{\alpha, \beta+1}^{(\gamma ; c)}(z ; p)+\alpha z \frac{d}{d z} E_{\alpha, \beta+1}^{(\gamma ; c)}(z ; p),
$$

where $p \geq 0, \operatorname{Re}(c)>\operatorname{Re}(\gamma)>0, \operatorname{Re}(\alpha)>0, \operatorname{Re}(\beta)>0$.

In the next theorem, we give the Mellin transform of the extended Mittag-Leffler function in terms of the Wright generalized hypergeometric function. Note that the Wright generalized hypergeometric function is defined by [17]

$$
\begin{aligned}
{ }_{p} \Psi_{q}(z) & ={ }_{p} \Psi_{q}\left[\begin{array}{l}
\left(a_{1}, A_{1}\right),\left(a_{2}, A_{2}\right), \ldots,\left(a_{p}, A_{p}\right) \\
\left(b_{1}, B_{1}\right),\left(b_{2}, B_{2}\right), \ldots,\left(b_{p}, B_{q}\right)
\end{array}, z\right] \\
& =\sum_{k=0}^{\infty} \frac{\prod_{j=1}^{p} \Gamma\left(a_{j}+A_{j} k\right)}{\prod_{j=1}^{q} \Gamma\left(b_{j}+B_{j} k\right)} \frac{z^{k}}{k !},
\end{aligned}
$$

where the coefficients $A_{i}(i=1, \ldots, p)$ and $B_{j}(j=1, \ldots, q)$ are positive real numbers such that

$$
1+\sum_{j=1}^{q} B_{j}-\sum_{i=1}^{p} A_{i} \geq 0 .
$$

Theorem 5 (Mellin transform) The Mellin transform of the extended Mittag-Leffler function is given by

$$
\begin{gathered}
\mathcal{M}\left\{E_{\alpha, \beta}^{(\gamma ; c)}(z ; p) ; s\right\}=\frac{\Gamma(s) \Gamma(c+s-\gamma)}{\Gamma(\gamma) \Gamma(c-\gamma)} 2_{2} \Psi_{2}\left[\begin{array}{cc}
(c, 1), & (\gamma+s, 1) \\
(\beta, \gamma), & (c+2 s, 1)
\end{array}, z\right] \\
(p \geq 0, \operatorname{Re}(c)>\operatorname{Re}(\gamma)>0, \operatorname{Re}(\alpha)>0, \operatorname{Re}(s)>0, \operatorname{Re}(\beta)>0),
\end{gathered}
$$

where ${ }_{2} \Psi_{2}$ is the Wright generalized hypergeometric function.

Proof Taking the Mellin transform of the extended Mittag-Leffler function, we have

$$
\mathcal{M}\left\{E_{\alpha, \beta}^{(\gamma ; c)}(z ; p) ; s\right\}=\int_{0}^{\infty} p^{s-1} E_{\alpha, \beta}^{(\gamma ; c)}(z ; p) d p .
$$

Using equation (6) in equation (13), we get

$$
\begin{aligned}
& \mathcal{M}\left\{E_{\alpha, \beta}^{(\gamma ; c)}(z ; p) ; s\right\} \\
& =\frac{1}{B(\gamma, c-\gamma)} \int_{0}^{\infty} p^{s-1}\left[\int_{0}^{1} t^{\gamma-1}(1-t)^{c-\gamma-1} e^{-\frac{p}{t(1-t)}}\right] E_{\alpha, \beta}^{(c)}(t z) d t d p .
\end{aligned}
$$

Interchanging the order of integrals in equation (14), which is valid because of the conditions in the statement of the Theorem 5 , we get

$$
\begin{aligned}
& \mathcal{M}\left\{E_{\alpha, \beta}^{(\gamma ; c)}(z ; p) ; s\right\} \\
& \quad=\frac{1}{B(\gamma, c-\gamma)} \int_{0}^{1}\left[t^{\gamma-1}(1-t)^{c-\gamma-1} E_{\alpha, \beta}^{(c)}(t z)\right] \int_{0}^{\infty} p^{s-1} e^{-\frac{p}{t(1-t)}} d p d t .
\end{aligned}
$$


Now taking $u=\frac{p}{t(1-t)}$ in equation (15) and using the fact that $\Gamma(s)=\int_{0}^{\infty} u^{s-1} e^{-u} d u$, we get

$$
\begin{aligned}
& \mathcal{M}\left\{E_{\alpha, \beta}^{(\gamma ; c)}(z ; p) ; s\right\} \\
& =\frac{\Gamma(s)}{B(\gamma, c-\gamma)} \int_{0}^{1} t^{\gamma+s-1}(1-t)^{c+s-\gamma-1} E_{\alpha, \beta}^{(c)}(t z) d t .
\end{aligned}
$$

Using the definition of Prabhakar's generalized Mittag-Leffler function $E_{\alpha, \beta}^{(c)}(t z)$ in equation (16), we get

$$
\begin{aligned}
& \mathcal{M}\left\{E_{\alpha, \beta}^{(\gamma ; c)}(z ; p) ; s\right\} \\
& =\frac{\Gamma(s)}{B(\gamma, c-\gamma)} \int_{0}^{1} t^{\gamma+s-1}(1-t)^{c+s-\gamma-1} \sum_{k=0}^{\infty} \frac{(c)_{k}(t z)^{k}}{\Gamma(\alpha k+\beta) k !} d t .
\end{aligned}
$$

Interchanging the order of summation and integration, which is valid for $\operatorname{Re}(c)>\operatorname{Re}(\gamma)>$ $0, \operatorname{Re}(s)>0, \operatorname{Re}(c-\gamma+s)>0, \operatorname{Re}(\alpha)>0, \operatorname{Re}(\beta)>0$, we get

$$
\begin{aligned}
& \mathcal{M}\left\{E_{\alpha, \beta}^{(\gamma ; c)}(z ; p) ; s\right\} \\
& =\frac{\Gamma(s)}{B(\gamma, c-\gamma)} \sum_{k=0}^{\infty} \frac{(c)_{k} z^{k}}{\Gamma(\alpha k+\beta) k !} \int_{0}^{1} t^{\gamma+k+s-1}(1-t)^{c+s-\gamma-1} d t .
\end{aligned}
$$

Using the Beta function in equation (18), we have

$$
\begin{aligned}
& \mathcal{M}\left\{E_{\alpha, \beta}^{(\gamma ; c)}(z ; p) ; s\right\} \\
& =\frac{\Gamma(s) \Gamma(c+s-\gamma)}{B(\gamma, c-\gamma)} \sum_{k=0}^{\infty} \frac{(c)_{k} z^{k}}{\Gamma(\alpha k+\beta) k !} \frac{\Gamma(\gamma+k+s) \Gamma(\gamma+s)}{\Gamma(\gamma+s) \Gamma(c+k+2 s)} .
\end{aligned}
$$

Considering that $(c)_{k}=\frac{\Gamma(c+k)}{\Gamma(c)}, B(\gamma, c-\gamma)=\frac{\Gamma(\gamma) \Gamma(c-\gamma)}{\Gamma(c)}$, and inserting equation (11) into equation (19), we get the result

$$
\begin{aligned}
\mathcal{M}\left\{E_{\alpha, \beta}^{(\gamma ; c)}(z ; p) ; s\right\} & =\frac{\Gamma(s) \Gamma(c+s-\gamma)}{B(\gamma, c-\gamma) \Gamma(c)} \sum_{k=0}^{\infty} \frac{z^{k}}{\Gamma(\alpha k+\beta) k !} \frac{\Gamma(\gamma+k+s) \Gamma(c+k)}{\Gamma(c+k+2 s)} \\
& =\frac{\Gamma(s) \Gamma(c+s-\gamma)}{\Gamma(\gamma) \Gamma(c-\gamma)}{ }_{2} \Psi_{2}\left[\begin{array}{c}
(c, 1), \quad(\gamma+s, 1) \\
(\beta, \alpha),(c+2 s, 1)
\end{array}\right] .
\end{aligned}
$$

Corollary 6 Taking $s=1$ in Theorem 5 , we get

$$
\int_{0}^{\infty} E_{\alpha, \beta}^{(\gamma ; c)}(z ; p) d p=\frac{\Gamma(c+1-\gamma)}{\Gamma(\gamma) \Gamma(c-\gamma)} 2 \Psi_{2}\left[\begin{array}{cc}
(c, 1), & (\gamma+1,1) \\
(\beta, \alpha), & (c+2,1)
\end{array}, z\right]
$$

Corollary 7 Taking the inverse Mellin transform on both sides of equation (12), we get the elegant complex integral representation

$$
E_{\alpha, \beta}^{(\gamma ; c)}(z ; p)=\frac{1}{2 \pi i \Gamma(\gamma) \Gamma(c-\gamma)} \int_{v-i \infty}^{v+i \infty} \Gamma(s) \Gamma(c+s-\gamma)_{2} \Psi_{2}\left[\begin{array}{cc}
(c, 1), & (\gamma+s, 1) \\
(\beta, \alpha), & (c+2 s, 1)
\end{array}\right], z p^{-s} d s
$$

where $v>0$. 


\section{Derivative properties of the extended Mittag-Leffler function}

The classical Riemann-Liouville fractional derivative of order $\mu$ is usually defined by

$$
D_{z}^{\mu}\{f(z)\}=\frac{1}{\Gamma(-\mu)} \int_{0}^{z} f(t)(z-t)^{-\mu-1} d t, \quad \operatorname{Re}(\mu)<0,
$$

where the integration path is a line from 0 to $z$ in the complex $t$-plane. For the case $m-1<$ $\operatorname{Re}(\mu)<m(m=1,2,3, \ldots)$, it is defined by

$$
\begin{aligned}
D_{z}^{\mu}\{f(z)\} & =\frac{d^{m}}{d z^{m}} D_{z}^{\mu-m}\{f(z)\} \\
& =\frac{d^{m}}{d z^{m}}\left\{\frac{1}{\Gamma(-\mu+m)} \int_{0}^{z} f(t)(z-t)^{-\mu+m-1} d t\right\} .
\end{aligned}
$$

The extended Riemann-Liouville fractional derivative operator was defined by Özarslan and Özergin as follows.

Definition 8 ([18]) The extended Riemann-Liouville fractional derivative is defined as

$$
D_{z}^{\mu, p}\{f(z)\}=\frac{1}{\Gamma(-\mu)} \int_{0}^{z} f(t)(z-t)^{-\mu-1} \exp \left(\frac{-p z^{2}}{t(z-t)}\right) d t, \quad \operatorname{Re}(\mu)<0, \operatorname{Re}(p)>0
$$

and for $m-1<\operatorname{Re}(\mu)<m(m=1,2,3, \ldots)$

$$
\begin{aligned}
D_{z}^{\mu, p}\{f(z)\} & =\frac{d^{m}}{d z^{m}} D_{z}^{\mu-m}\{f(z)\} \\
& =\frac{d^{m}}{d z^{m}}\left\{\frac{1}{\Gamma(-\mu+m)} \int_{0}^{z} f(t)(z-t)^{-\mu+m-1} \exp \left(\frac{-p z^{2}}{t(z-t)}\right) d t\right\},
\end{aligned}
$$

where the path of integration is a line from 0 to $z$ in the complex $t$-plane. For the case $p=0$, we obtain the classical Riemann-Liouville fractional derivative operator.

We begin by the following theorem.

Theorem 9 Let $p \geq 0, \operatorname{Re}(\mu)>\operatorname{Re}(\lambda)>0, \operatorname{Re}(\alpha)>0, \operatorname{Re}(\beta)>0$. Then

$$
D_{z}^{\lambda-\mu, p}\left\{z^{\lambda-1} E_{\alpha, \beta}^{(c)}(z)\right\}=\frac{z^{\mu-1} B(\lambda, c-\lambda)}{\Gamma(\mu-\lambda)} E_{\alpha, \beta}^{(\lambda ; \mu)}(z ; p)
$$

Proof Replacing $\mu$ by $\lambda-\mu$ in the definition of the extended fractional derivative operator (20), we get

$$
\begin{aligned}
& D_{z}^{\lambda-\mu, p}\left\{z^{\lambda-1} E_{\alpha, \beta}^{(c)}(z)\right\} \\
& \quad=\frac{1}{\Gamma(\mu-\lambda)} \int_{0}^{z} t^{\lambda-1} E_{\alpha, \beta}^{(c)}(t)(z-t)^{-\lambda+\mu-1} \exp \left\{\frac{-p z^{2}}{t(z-t)}\right\} d t \\
& \quad=\frac{z^{-\lambda+\mu-1}}{\Gamma(\mu-\lambda)} \int_{0}^{z} t^{\lambda-1} E_{\alpha, \beta}^{(c)}(t)\left(1-\frac{t}{z}\right)^{-\lambda+\mu-1} \exp \left\{\frac{-p z^{2}}{t(z-t)}\right\} d t .
\end{aligned}
$$


Taking $u=\frac{t}{z}$ in equation (21), we get

$$
\begin{aligned}
& D_{z}^{\lambda-\mu, p}\left\{z^{\lambda-1} E_{\alpha, \beta}^{(c)}(z)\right\} \\
& \quad=\frac{z^{\mu-1}}{\Gamma(\mu-\lambda)} \int_{0}^{1} u^{\lambda-1}(1-u)^{-\lambda+\mu-1} \exp \left\{\frac{-p}{u(1-u)}\right\} E_{\alpha, \beta}^{(c)}(u z) d u .
\end{aligned}
$$

Comparing this result with equation (6), we get

$$
D_{z}^{\lambda-\mu, p}\left\{z^{\lambda-1} E_{\alpha, \beta}^{(c)}(z)\right\}=\frac{z^{\mu-1} B(\lambda, c-\lambda)}{\Gamma(\mu-\lambda)} E_{\alpha, \beta}^{(\lambda ; \mu)}(z ; p) .
$$

Whence the result.

In the following theorem, we give the derivative properties of the extended MittagLeffler function.

Theorem 10 For the extended Mittag-Leffler function, we have the following derivative formula:

$$
\frac{d^{n}}{d z^{n}}\left\{E_{\alpha, \beta}^{(\gamma ; c)}(z ; p)\right\}=(c)_{n} E_{\alpha, \beta+n \alpha}^{(\gamma+n ; c+n)}(z ; p), \quad n \in \mathbb{N}
$$

Proof Taking the derivative with respect to $z$ in equation (6), we get

$$
\frac{d}{d z}\left\{E_{\alpha, \beta}^{(\gamma ; c)}(z ; p)\right\}=c E_{\alpha, \beta+\alpha}^{(\gamma+1 ; c+1)}(z ; p) .
$$

Again taking the derivative with respect to $z$ in equation (24), we get

$$
\frac{d^{2}}{d z^{2}}\left\{E_{\alpha, \beta}^{(\gamma ; c)}(z ; p)\right\}=c(c+1) E_{\alpha, \beta+2 \alpha}^{(\gamma+2 ; c+2)}(z ; p) .
$$

Continuing the repetition of this procedure $n$ times, we get the desired result.

Theorem 11 For the extended Mittag-Leffler function, the following differentiation formula holds:

$$
\frac{d^{n}}{d z^{n}}\left\{z^{\beta-1} E_{\alpha, \beta}^{(\gamma ; c)}\left(\lambda z^{\alpha} ; p\right)\right\}=z^{\beta-n-1} E_{\alpha, \beta-n}^{(\gamma ; c)}\left(\lambda z^{\alpha} ; p\right) .
$$

Proof In equation (23), replace $z$ by $\lambda z^{\alpha}$ and multiply $z^{\beta-1}$, then taking the $z$-derivative $n$ times, we get the result.

Theorem 12 For the extended Mittag-Leffler function, the following differentiation formula holds:

$$
\frac{d^{n}}{d p^{n}}\left\{E_{\alpha, \beta}^{(\gamma ; c)}(z ; p)\right\}=(-1)^{n} \frac{\Gamma(\gamma-n) \Gamma(c-\gamma-n) \Gamma(c)}{\Gamma(c-2 n) \Gamma(\gamma) \Gamma(c-\gamma)} E_{\alpha, \beta}^{(\gamma-n ; c-2 n)}(z ; p) .
$$

Proof Taking the $p$-derivative $n$ times in equation (6), we get the result. 


\section{Relations between the extended Mittag-Leffler function with Laguerre polynomial and Whittaker function}

In this section, we give a representation of the extended Mittag-Leffler function in terms of Laguerre polynomials and Whittaker's function.

Theorem 13 For the extended Mittag-Leffler function, we have

$$
\begin{aligned}
& \exp (2 p) E_{\alpha, \beta}^{(\gamma ; c)}(z ; p) \\
& \quad=\frac{1}{B(\gamma, c-\gamma)} \sum_{m, n, k=0}^{\infty} \frac{L_{m}(p) L_{n}(p)(c)_{k}}{\Gamma(\alpha k+\beta) k !} z^{k} B(m+k+\gamma+1, n+c-\gamma+1)
\end{aligned}
$$

where $\operatorname{Re}(c)>\operatorname{Re}(\gamma)>0, \operatorname{Re}(\alpha)>0, \operatorname{Re}(\beta)>0$.

Proof We start by recalling the useful identity used in [18]

$$
\exp \left(\frac{-p}{t(1-t)}\right)=\exp (-2 p) \sum_{m, n=0}^{\infty} L_{n}(p) L_{m}(p) t^{m+1}(1-t)^{n+1} ; \quad 0<t<1
$$

Using equation (26) in equation (6), we get

$$
\begin{aligned}
E_{\alpha, \beta}^{(\gamma ; c)}(z ; p)= & \frac{1}{B(\gamma, c-\gamma)} \int_{0}^{1} t^{\gamma-1}(1-t)^{c-\gamma-1} \exp (-2 p) \\
& \times \sum_{m, n=0}^{\infty} L_{n}(p) L_{m}(p) t^{m+1}(1-t)^{n+1} E_{\alpha, \beta}^{c}(t z) d t .
\end{aligned}
$$

Now, taking into account the series expansion of Prabhakar's generalized Mittag-Leffler's function $E_{\alpha, \beta}^{c}(t z)$ in equation (27), we have

$$
\begin{aligned}
E_{\alpha, \beta}^{(\gamma ; c)}(z ; p)= & \frac{\exp (-2 p)}{B(\gamma, c-\gamma)} \int_{0}^{1} t^{\gamma-1}(1-t)^{c-\gamma-1} \\
& \times \sum_{m, n=0}^{\infty} L_{n}(p) L_{m}(p) t^{m+1}(1-t)^{n+1} \sum_{k=0}^{\infty} \frac{(c)_{k}(t z)^{k}}{\Gamma(\alpha k+\beta) k !} d t \\
= & \frac{\exp (-2 p)}{B(\gamma, c-\gamma)} \int_{0}^{1} t^{\gamma-1}(1-t)^{c-\gamma-1} \\
& \times \sum_{m, n, k=0}^{\infty} \frac{L_{n}(p) L_{m}(p)(c)_{k}}{\Gamma(\alpha k+\beta) k !} t^{m+k+1}(1-t)^{n+1} z^{k} d t .
\end{aligned}
$$

Interchanging the order of integration and summation in equation (28), which can be done under the assumptions of the theorem, we have

$$
\begin{aligned}
E_{\alpha, \beta}^{(\gamma ; c)}(z ; p)= & \frac{\exp (-2 p)}{B(\gamma, c-\gamma)} \sum_{m, n, k=0}^{\infty} \frac{L_{n}(p) L_{m}(p)(c)_{k}}{\Gamma(\alpha k+\beta) k !} z^{k} \\
& \times B(m+k+\gamma+1, n+c-\gamma+1) .
\end{aligned}
$$

Multiplying both sides of equation (29) by $\exp (2 p)$, we get the result. 
In the following theorem, we give the extended Mittag-Leffler function in terms of Whittaker's function.

Theorem 14 For the extended Mittag-Leffler function we have

$$
\exp \left(\frac{3 p}{2}\right) E_{\alpha, \beta}^{(\gamma ; c)}(z ; p)=\frac{\Gamma(c-\gamma+1)}{B(\gamma, c-\gamma)} \sum_{m, k=0}^{\infty} \frac{L_{m}(p)(c) k}{\Gamma(\alpha k+\beta) k !} p^{\frac{m+k+\gamma-1}{2}} W_{\frac{\gamma-2 c-m-1}{2}, \frac{m+k+\gamma}{2}}(p) .
$$

Proof Considering the following equality:

$$
\exp \left(\frac{-p}{t(1-t)}\right)=\exp \left(\frac{-p}{1-t}\right) \exp \left(\frac{-p}{t}\right)
$$

and using the generating function of the Laguerre polynomials, we get

$$
\exp \left(\frac{-p}{t(1-t)}\right)=\exp (-p) \exp \left(\frac{-p}{t}\right)(1-t) \sum_{m=0}^{\infty} L_{m}(p) t^{m}
$$

Taking equation (30) into account in equation (6), we have

$$
\begin{aligned}
E_{\alpha, \beta}^{(\gamma ; c)}(z ; p)= & \frac{1}{B(\gamma, c-\gamma)} \int_{0}^{1} t^{\gamma-1}(1-t)^{c-\gamma-1} e^{-\frac{p}{t(1-t)}} E_{\alpha, \beta}^{(c)}(t z) d t \\
= & \frac{1}{B(\gamma, c-\gamma)} \int_{0}^{1} t^{\gamma-1}(1-t)^{c-\gamma-1} \exp (-p) \exp \left(\frac{-p}{t}\right) \\
& \times(1-t) \sum_{m=0}^{\infty} L_{m}(p) t^{m} E_{\alpha, \beta}^{(c)}(t z) d t .
\end{aligned}
$$

By use of Prabhakar's generalized Mittag-Leffler function $E_{\alpha, \beta}^{(c)}(t z)$ in equation (31), we get

$$
\begin{aligned}
& E_{\alpha, \beta}^{(\gamma ; c)}(z ; p) \\
& \quad=\frac{\exp (-p)}{B(\gamma, c-\gamma)} \int_{0}^{1} t^{\gamma-1}(1-t)^{c-\gamma} \exp \left(\frac{-p}{t}\right) \sum_{m=0}^{\infty} L_{m}(p) t^{m} \sum_{k=0}^{\infty} \frac{(c)_{k} t^{k} z^{k}}{\Gamma(\alpha k+\beta) k !} d t .
\end{aligned}
$$

Interchanging the order of summation and integration in equation (32), we get

$$
\begin{aligned}
& E_{\alpha, \beta}^{(\gamma ; c)}(z ; p) \\
& \quad=\frac{\exp (-p)}{B(\gamma, c-\gamma)} \sum_{m, k=0}^{\infty} \frac{L_{m}(p)(c)_{k} z^{k}}{\Gamma(\alpha k+\beta) k !} \int_{0}^{1} t^{m+k+\gamma-1}(1-t)^{c-\gamma} \exp \left(\frac{-p}{t}\right) d t .
\end{aligned}
$$

Finally, using the following integral representation [19]:

$$
\begin{aligned}
& \int_{0}^{1} t^{\mu-1}(1-t)^{v-1} \exp \left(\frac{-p}{t}\right) d t=\Gamma(v) p^{\frac{\mu-1}{2}} \exp \left(\frac{-p}{2}\right) W_{\frac{1-\mu-2 v}{2}, \frac{\mu}{2}}(p) \\
& (\operatorname{Re}(v)>0, \operatorname{Re}(p)>0)
\end{aligned}
$$

in equation (33), we get the result. 


\section{Competing interests}

The authors declare that they have no competing interests.

\section{Authors' contributions}

All authors completed the paper together. All authors read and approved the final manuscript.

Received: 5 November 2013 Accepted: 13 December 2013 Published: 20 Feb 2014

\section{References}

1. Camargo, RF, Capelas de Oliveira, E, Vaz, J: On the generalized Mittag-Leffler function and its application in a fractional telegraph equation. Math. Phys. Anal. Geom. 15(1), 1-16 (2012)

2. Samko, SG, Kilbas, AA, Marichev, OI: Fractional Integrals and Derivatives: Theory and Applications. Gordon \& Breach, New York (1993)

3. Haubold, HJ, Mathai, AM, Saxena, RK: Mittag-Leffler functions and their applications. J. Appl. Math. 2011, Article ID 298628 (2011). doi:10.1155/2011/298628

4. Soubhia, AL, Camargo, RF, Capelas de Oliveira, E, Vaz, J: Theorem for series in three-parameter Mittag-Leffler function. Fract. Calc. Appl. Anal. 13, 1-12 (2010)

5. Mittag-Leffler, GM: Une généralisation de l'intégrale de Laplace-Abel. C. R. Acad. Sci., Ser. II 137, $537-539$ (1903)

6. Mittag-Leffler, GM: Sur la nouvelle fonction $E_{\alpha}(x)$. C. R. Acad. Sci. 137, 554-558 (1903)

7. Mittag-Leffler, GM: Sur la représentation analytique d'une fonction monogéne (cinquiéme note). Acta Math. 29(1), 101-181 (1905)

8. Wiman, A: Über der Fundamentalsatz in der Theorie der Funktionen $E_{\alpha}(x)$. Acta Math. 29, 191-201 (1905)

9. Wiman, A: Über die Nullstellen der Funktionen $E_{\alpha}(x)$. Acta Math. 29, 217-234 (1905)

10. Agarwal, RP: A propos d'une note de M. Pierre Humbert. C. R. Séances Acad. Sci. 236, 2031-2032 (1953)

11. Humbert, P, Agarwal, RP: Sur la fonction de Mittag-Leffler et quelques unes de ses generalizations. Bull. Sci. Math., Ser. II 77, 180-185 (1953)

12. Prabhakar, TR: A singular integral equation with a generalized Mittag-Leffler function in the kernel. Yokohama Math. J. 19, 7-15 (1971)

13. Kurulay, M, Bayram, M: Some properties of the Mittag-Leffler functions and their relation with the Wright function. Adv. Differ. Equ. 2012, 178 (2012)

14. Mainardi, F: On some properties of the Mittag-Leffler function, $E_{\alpha}(t z)$, completely monotone for $t>0$ with $0<\alpha<1$. arXiv:1305.0161

15. Chaudhry, MA, Qadir, A, Srivastava, HM, Paris, RB: Extended hypergeometric and confluent hypergeometric functions. Appl. Math. Comput. 159, 589-602 (2004)

16. Chaudhry, MA, Zubair, SM: On a Class of Incomplete Gamma Functions with Applications (2002)

17. Srivastava, HM, Manocha, HL: A Treatise on Generating Functions. Halsted, New York (Ellis Horwood, Chichester) (1984)

18. Özarslan, MA, Özergin, E: Some generating relations for extended hypergeometric functions via generalized fractional derivative operator. Math. Comput. Model. 52, 1825-1833 (2010)

19. Özarslan, MA: Some remarks on extended hypergeometric, extended confluent hypergeometric and extended Appell's functions. J. Comput. Anal. Appl. 14(6), 1148-1153 (2012)

10.1186/1029-242X-2014-85

Cite this article as: Özarslan and Yılmaz: The extended Mittag-Leffler function and its properties. Journal of Inequalities and Applications 2014, 2014:85

\section{Submit your manuscript to a SpringerOpen ${ }^{\circ}$ journal and benefit from:}

- Convenient online submission

- Rigorous peer review

- Immediate publication on acceptance

- Open access: articles freely available online

- High visibility within the field

- Retaining the copyright to your article 